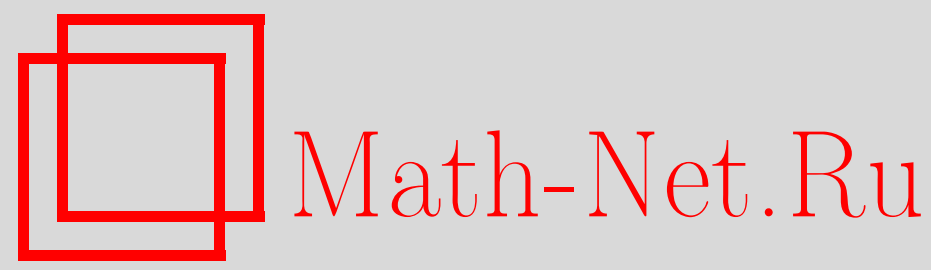

А. А. Борисенко, О компактных подмногообразиях неположительной внешней кривизны в римановом пространстве, Матем. заметки, 1996, том 60, выпуск 1, 3-10

DOI: https://doi.org/10.4213/mzm1798

Использование Общероссийского математического портала MathNet.Ru подразумевает, что вы прочитали и согласны с пользовательским соглашением

http://www . mathnet.ru/rus/agreement

Параметры загрузки:

IP: 54.196 .121 .252

26 апреля 2023 г., 13:27:16

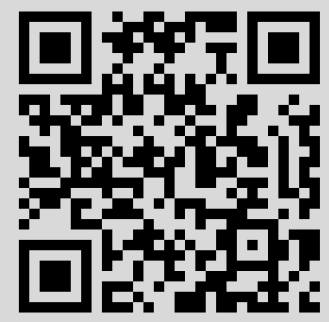




\section{О КОМПАКТНЫХ ПОДМНОГООБРАЗИЯХ НЕПОЛОЖИТЕЛЬНОЙ ВНЕШНЕЙ КРИВИЗНЫ В РИМАНОВОМ ПРОСТРАНСТВЕ}

\section{А. А. Борисенко}

В работе [1] были доказаны теоремы, в которых выясняется, когда компактные поверхности неположительной внешней кривизны в римановом пространстве являются вполне геодезическими подмногообразиями, изометричными стандартной сфере и когда объемлющее пространство изометрично стандартной сфере.

Однако, в этих теоремах сушественно использовалось, что в точках поверхности $F^{l}$ для объемлющего риманова многообразия $M^{l+p}$ должновыполняться условие

$$
R(x, y) \xi=0,
$$

где $x, y$ - произвольные касательные векторы к подмногообразию $F^{l}, \xi$ нормальный вектор, $R$ - оператор кривизны для $M^{l+p}$.

Мы докажем аналог этих теорем без требования (1), но с более сильными ограничениями на коразмерность.

Пусть $\rho(l-p)-1$ - максимальное число непрерывных линейно независимых в каждой точке векторных полей на сфере $S^{l-p}$

$$
\begin{gathered}
\nu(l)=\max \{t, t<\rho(l-t)\}, \\
\nu(l)<2 \log _{2}(l)+1, \quad \nu\left(2^{b}\right)=0 .
\end{gathered}
$$

Теорема 1. Пусть $F^{l}$ - компактная поверхность в полном римановом пространстве $M^{l+p}$, секционная кривизна которого удовлетворяет неравенству $\gamma_{2 / M} \geqslant 1$.

Пусть для поверхности выполняется одно из следующих условий:

1) $\gamma_{2 / F} \leqslant 1, p \leqslant-3 / 4+\sqrt{9 / 16+l / 2}$

2) $0<\gamma_{2 / F} \leqslant 1, p<-1 / 2+\sqrt{1 / 4+l-\nu(l)}$. 
Тогда поверхность есть вполне геодезическое подмногообразие, локально изометричное единичной сфере.

Полнота объемлющего пространства используется только в случае 1). Если $F^{l}$ односвязно, то поверхность глобально изометрична единичной cфepe.

В теореме 4 работы [1] при выполнении условия 4 теорема сформулирована неточно. Из него только следует, что поверхность будет изометрична единичной сфере, но еще не следует вполне геодезичность.

При более строгих ограничениях на кривизну $M^{l+p}$ мы получим

ТЕОрема 2. Пусть $F^{l}$ есть компактная поверхность в полном односвязном римановом пространстве $M^{l+p}$. Если

$$
\frac{9}{4} \geqslant \gamma_{2 / M} \geqslant 1, \quad \gamma_{2 / F} \leqslant 1, \quad p<\frac{1}{2}+\sqrt{\frac{1}{4}+l-1},
$$

то риманово многообразие $M^{l+p}$ глобально изометрично единичной cфере.

Из теорем 1, 2 непосредственно следует

ТЕОрема 3. Пусть $F^{l}$ есть компактная поверхность в полном односвязном римановом пространстве $M^{l+p}$, кривизна которого удовлетворяет неравенству $9 / 4 \geqslant \gamma_{2}(M) \geqslant 1$. Если выполняется одно из условий теоремы 1 , то обвемлющее пространство глобально изометрично единичной сфере, и поверхность есть вполне геодезическая большая сфера.

Если выполняются условия теоремы 2 , то утверждение теоремы 3 справедливо при $l \geqslant 19$.

Внешний нуль-индекс $\nu(Q)$ точки $Q \in F^{l}$ - это максимальная размерность нулевого подпространства $L(Q) \subset T_{Q} F^{l}$ такого, что для любого $y \in L(Q)$ выполняется равенство

$$
A(Q, n) y=0
$$

для каждой нормали $n$ в точке $Q$, где $A(Q, n)$ - матрица второй квадратичной формы $F^{l}$ относительно нормали $n ; \nu^{*}(Q)$ есть минимальный нуль-индекс среди точек близких $Q, \nu^{*}(Q) \leqslant \nu(Q)$.

Для поверхностей неположительной внешней кривизны, т.е. для котоpыx

$$
\gamma_{2 / F^{l}}-\gamma_{2 / M^{l+p}} \leqslant 0,
$$

имеет место оценка нуль-индекса $\nu$ для поверхностей неположительной внешней кривизны. 
ЛЕмма 1 [2]. Справедливо неравенство

$$
\nu \geqslant l-p(p+1)
$$

Пусть индексы меняются в следующих пределах:

$$
\begin{gathered}
a, b, c=1, \ldots, \nu, \quad i, j, k=1, \ldots, l, \\
A, B, C, D=1, \ldots, l+p, \quad \alpha, \beta, \gamma, \sigma=l+1, \ldots, l+p .
\end{gathered}
$$

ЛЕмма 2. Пусть в точке $Q$ риманова многообразия $M^{l+p}$ секиионная кривизна достигает максимального значения $k$ на площадках $\sigma$, проходящих через вектор $x \in L(Q)$, и любой вектор y $\in T_{Q}^{l}$, $L(Q) \subset T_{Q}^{l} \subset T_{Q} M^{l+p}$, әде $L(Q), T_{Q}^{l}$ - подпространства касательного пространства $T_{Q} M^{l+p}$.

Тогда

1) $(R(x, y) y)^{\perp}=0$, где $x \in L(Q), y \in T_{Q}^{l}, R(x, y)$ - оператор кривизны многообразия $M^{l+p}, \perp$-ортогональная проекиия на подпространство, ортогональное $T_{Q}^{l}$;

2) $R(x, y) x=-k\langle x, x\rangle y$, где $x \in L(Q), y \in L^{\perp}(Q) \subset T_{Q}^{l}$.

ДокаЗАТЕЛЬСтво. Секционная кривизна $M^{l+p}$ в точке $Q$ в направлении векторов $x, y \in T_{Q}^{l}$ равна

$$
k(x, y)=\frac{R_{A B C D} x^{A} x^{C} y^{B} y^{D}}{\left(g_{A C} g_{B D}-g_{A D} g_{B C}\right) x^{A} x^{C} y^{B} y^{D}} .
$$

Возьмем координаты в $M^{l+p}$ так, чтобы в точке $Q g_{A B}=\delta_{A B}, y \in T_{Q}^{l}-$ произвольный фиксированньй вектор, $f$ будем рассматривать как функцию $x^{A}$.

В экстремальной точке $x f=k$ и

$$
\frac{\partial f}{\partial x^{C}}=2\left[R_{A B C D} x^{A} y^{B} y^{D}-k\left(g_{A C} g_{B D}-g_{A D} g_{B C}\right) x^{A} y^{B} y^{D}\right]=0 .
$$

Это справедливо для $x \in L(Q), y \in T_{Q}^{l}$.

В операторном виде (2) запишется как

$$
R(x, y) y=k(\langle y, y\rangle x-\langle x, y\rangle y) .
$$

Отсюда непосредственно следует, что

$$
(R(x, y) y)^{\perp}=0
$$


при $x, y \in T_{Q}^{l}$.

Если $y \in L(Q)$, то экстремальное значение функция $f$ принимает также на $x \in L^{\perp}(Q)$, и $\left(2^{\prime}\right)$ принимает вид

$$
R(y, x) y=-k\langle y, y\rangle x
$$

Переобозначив $x$ на $y$ и наоборот, мы получим нужное соотношение.

Так как

$$
R(x, y+z)(y+z)=R(x, y) y+R(x, z) z+R(x, y) z+R(x, z) y
$$

то для $y, z \in T_{Q}^{l}, x \in L(Q)$ из (3) следует, что

$$
\langle R(x, y) z+R(x, z) y, n\rangle=0
$$

для любого вектора $n$, ортогонального $T_{Q}^{l}$.

ЛЕмма 3. Пусть $F^{l}$-регулярная класса $C^{3}$ l-мерная поверхность в римановом пространстве $M^{l+p}$, внешний нуль-индекс которой $\nu(Q)$ постоянен в окрестности точки $Q_{0}, \nu(Q)=\nu\left(Q_{0}\right)=\nu_{0}$, $L(Q) \subset T_{Q} F^{l}$ - нулевое подпространство.

Пусть в точках поверхности для тензора кривизны обгемлющего пространства выполняется условие

$$
R(x, y) y^{\perp}=0
$$

где $x \in L(Q), y \in T_{Q} F^{l}, \perp$ - ортогональная проекиия вектора на нормальное пространство $N_{Q} F^{l}$.

Тогда распределение $L(Q)$ голономно, слои являются вполне геодезическими подмногообразиями, $R^{\nu_{0}}(Q) \subset M^{l+p}, \nu(Q)=\nu\left(Q_{0}\right)$ для внутренних точек $R^{k}\left(Q_{0}\right), \nu^{*}(Q) \leqslant \nu\left(Q_{0}\right)$ для граничных точек $R^{k}\left(Q_{0}\right)$. Если поверхность полная $и \nu\left(Q_{0}\right)=\min _{Q \in F^{l}} \nu(Q)$, то $R^{k}\left(Q_{0}\right)$ есть полное риманово многообразие.

ДокАзАТЕЛьство. Выберем так параметризацию поверхности и нормальные векторные поля $n_{\alpha}$, чтобы в точке $Q_{0}$ метрический тензор поверхности

$$
g_{i j}=\delta_{i j}, \quad \frac{\partial g_{i j}}{\partial u_{k}}=0,
$$

коэффициенты кручения

$$
\mu_{\alpha \beta, i}=0 \text {. }
$$

В точке $Q_{0}$ касательные векторы $v_{i}$ имеют координаты

$$
v_{i}^{A}=\frac{\partial v^{A}}{\partial u_{i}}=\delta_{i}^{A}(z),
$$


где $v^{A}=v^{A}\left(u^{1}, \ldots, u^{l}\right)$ - параметризация поверхности; единичные векторы ортонормированного поля нормалей $n_{\alpha}^{B}=\delta_{\alpha}^{B}$, пространство $L(Q)$ натянуто на $\nu$ первых векторов $\nu_{a}=\left(v_{a}^{A}\right)$.

Тогда в точке $Q_{0}$ уравнения Кодацши для вторых квадратичных форм $A_{i j}^{\alpha}$ запишутся так [3]

$$
\begin{aligned}
& \frac{\partial A_{i j}^{\alpha}}{\partial u^{a}}-\frac{\partial A_{i a}^{\alpha}}{\partial u^{j}}=R_{i \alpha j a} \\
& \frac{\partial A_{i j}^{\alpha}}{\partial u^{a}}-\frac{\partial A_{j a}^{\alpha}}{\partial u^{i}}=R_{j \alpha i a} .
\end{aligned}
$$

Но, так как из (4) $R_{i \alpha j a}=R_{i \alpha i a}=0$, то

$$
\frac{\partial A_{i j}^{\alpha}}{\partial u^{a}}=\frac{1}{2}\left(\frac{\partial A_{i a}^{\alpha}}{\partial u^{j}}+\frac{\partial A_{j a}^{\alpha}}{\partial u^{i}}\right)
$$

Пусть $x(Q), y(Q)$ - регулярные векторные поля на $F^{l}$, принадлежащие распределению, т.е.

$$
A_{i j}^{\alpha} x^{i}=0, \quad A_{i j}^{\alpha} y^{j}=0 .
$$

В точке $Q_{0}$ из выбора системы координат следует, что

$$
x^{i}=0, \quad y^{i}=0, \quad \text { если } i>\nu .
$$

Продифференцируем (6) по $u^{k}$

$$
A_{i j}^{\alpha} \frac{\partial x^{i}}{\partial u^{k}}+\frac{\partial A_{i j}^{\alpha}}{\partial u^{k}} x^{i}=0, \quad A_{j i}^{\alpha} \frac{\partial y^{i}}{\partial u^{k}}+\frac{\partial A_{j i}^{\alpha}}{\partial u^{k}} y^{i}=0
$$

Умножим первое уравнение на $y^{k}$, второе - на $x^{k}$ и просуммируем. Тогда

$$
A_{i j}^{\alpha} \frac{\partial x^{i}}{\partial u^{k}} y^{k}+\frac{\partial A_{i j}^{\alpha}}{\partial u^{k}} x^{i} y^{k}=0, \quad A_{j i}^{\alpha} \frac{\partial y^{i}}{\partial u^{k}} x^{k}+\frac{\partial A_{j i}^{\alpha}}{\partial u^{k}} y^{i} x^{k}=0 \text {. }
$$

Из (7) следует, что в точке $Q_{0}$

$$
A_{i j}^{\alpha} \frac{\partial x^{i}}{\partial u^{a}} y^{a}+\frac{\partial A_{i j}^{\alpha}}{\partial u^{a}} x^{i} y^{a}=0
$$

Подставив (5) в (8), получим

$$
A_{i j}^{\alpha} \frac{\partial x^{i}}{\partial u^{a}} y^{a}+\frac{1}{\alpha^{2}}\left(\frac{\partial A_{i a}^{\alpha}}{\partial u^{j}} x^{i} y^{a}+\frac{\partial A_{j a}^{\alpha}}{\partial u^{i}} x^{i} y^{a}\right)=0
$$


Из (6)

$$
A_{i k}^{\alpha} x^{i} y^{k}=0 .
$$

Продифференцируем по $u^{j}$, получим

$$
\frac{\partial A_{i k}^{\alpha}}{\partial u^{j}} x^{i} y^{k}+A_{i k}^{\alpha} \frac{\partial x^{i}}{\partial u^{j}} y^{k}+A_{i k}^{\alpha} \frac{\partial y^{k}}{\partial u^{j}} x^{i}=0 .
$$

Два последних слагаемых равны нулю в силу (6). Поэтому в точке $Q_{0}$

$$
\frac{\partial A_{i a}^{\alpha}}{\partial u^{j}} x^{i} y^{a}=0 .
$$

Подставив (10) в (9), а также учитьвая, что в точке $Q_{0}$

$$
\frac{\partial A_{j a}^{\alpha}}{\partial u^{i}} x^{i} y^{a}=-A_{j k} \frac{\partial y^{k}}{\partial u^{a}} x^{a}
$$

мы получим

$$
2 A_{i j}^{\alpha} \frac{\partial x^{i}}{\partial u^{a}} y^{a}-A_{j k} \frac{\partial y^{k}}{\partial u^{a}} x^{a}=0 .
$$

Аналогично,

$$
2 A_{j k}^{\alpha} \frac{\partial y^{k}}{\partial u^{a}} x^{a}-A_{i j}^{\alpha} \frac{\partial x^{i}}{\partial u^{a}} y^{a}=0 .
$$

Отсюда

$$
A_{i j}^{\alpha} \frac{\partial x^{i}}{\partial u^{a}} y^{a}=0, \quad A_{j k}^{\alpha} \frac{\partial y^{k}}{\partial u^{a}} x^{a}=0 .
$$

С учетом выбора системы координат в точке $Q_{0}$

$$
\nabla_{x} y=\left(\frac{\partial y^{i}}{\partial u^{a}} x^{a}\right) v_{i}, \quad \nabla_{y} x=\left(\frac{\partial x^{i}}{\partial u^{a}} y^{a}\right) v_{i},
$$

где $\nabla$ - ковариантная производная в метрикеповерхности. Поэтому из (11) следует, что $\nabla_{x} y, \nabla_{y} x \subset L(Q)$. Так как скобка Ли векторных полей

$$
[x, y]=\nabla_{y} x-\nabla_{x} y
$$

также принадлежит $L(Q)$, то распределение $L(Q)$ голономно. Так как $\nabla_{x} y \subset L(Q)$, то слои являются вполне геодезическими подмногообразиями объемлющего пространства.

Дальнейшее доказательство проходит аналогично доказательству теоремы 1 из [1] или теоремы из [4], где доказывается аналогичное утверждение при условии, что вдоль поверхности

$$
\langle R(x, y) z, n\rangle=0,
$$

где $x, y, z \subset T_{Q} F^{l}, n$-произвольный нормальньй вектор.

Поверхность $F^{l}$ риманова пространства $M^{l+p}$ мы будем назьвать линейчатой, если на ней имеется $C^{1}$ слоение $\{L\}$, состоящее из вполне геодезических подмногообразий объемлюшего пространства. 
ЛЕмма 4. Пусть $F^{l} \subset M^{l+p}$ - линейчатая поверхность и вдоль полной образующей $L_{0} \in\{L\}$

$$
R(x, y) x=-k y, \quad k(x, y)>0
$$

где $k=$ const $>0, x \in T L_{0}, y \in T L_{0}^{\perp},\langle x, x\rangle=1$. Тогда размерность образующей $\nu<\nu(l)$.

ДоКАЗАТЕЛЬСТВО ТЕОРЕМЫ 1 . Поверхность $F^{l}$ по условиям теоремы является поверхностью неположительной внешней кривизны и нуль-индекс $\nu(Q)$ в силу ограничения на коразмерность по лемме 1 соответственно удовлетворяет неравенствам:

1) $\nu \geqslant(l+p) / 2$

2) $\nu>\nu(l)$.

Из формулы Гаусса непосредственно следует, что для любой двумерной плоскости $\sigma \subset T_{Q} F^{l}$, проходящей через вектор $x \in L(Q)$,

$$
\gamma_{2 / F^{l}}=\gamma_{2 / M^{l+p}}
$$

Но, так как $\gamma_{2 / M^{l+p}} \geqslant 1, \gamma_{2 / F^{l+p}} \leqslant 1$, то $\gamma_{2 / M^{l+p}}=1$, и секционная кривизна $M^{l+p}$ достигает на этой площадке минимума. Тогда из леммы 1 следует, что распределение $L(Q)$ удовлетворяет условиям леммы 2. Возьмем точку $Q_{0} \in F^{l}$, для которой $\nu\left(Q_{0}\right)=\min _{Q \in F^{l}} \nu(Q)$. Тогда в окрестности $Q_{0} \nu(Q)$ постоянно, и мы имеем дифференцируемое распределение. По лемме 3 через точки этой окрестности проходят полные вполне геодезические подмногообразия объемлющего пространства, которые расслаивают поверхность $F^{l}$. При этом различные слои не пересекаются. В случае 1) $\nu \geqslant(l+p) / 2$, и так как кривизна $M^{l+p} \geqslant 1$, то они должны по теореме Франкеля пересекаться [5]. Поэтому $\nu=l$ и поверхность $F^{l}$ есть вполне геодезическое подмногообразие, локально изометричное единичной сфере. В случае 2) $\nu>\nu(l)$. Но для поверхности вьполняются условия леммы 4. Это следует из лемм 2,3 . Поэтому $\nu=l$.

ДоКАЗАТЕЛЬСТВо ТЕОРЕМЫ 2. Из ограничения на коразмерность и леммы 1 следует, что $\nu \geqslant 2$. Так как для поверхности выполняются условия леммы 3 , то поверхность $F^{l}$ содержит полное вполне геодезическое подмногообразие с кривизной 1.

И дальнейшее доказательство совпадает с доказательством теоремы 5 из [1]. В силу односвязности $M^{l+p}$ и ограничений на кривизну $R^{\nu}$ есть односвязное подмногообразие, глобально изометричное единичной сфере. Возьмем на ней замкнутую геодезическую длины $2 \pi$, которая является замкнутой геодезической $M^{l+p}$. Так как кривизна $M^{l+p}$ есть $\gamma_{2} \leqslant 9 / 4$, то отрезок этой геодезической длины $2 \pi / 3$ является кратчайшим. Мы имеем треугольник, стороны которого являются кратчайшими, и сумма длин 
этих сторон равна $2 \pi$. Тогда по экстремальной теореме Топоногова [6] риманово пространство $M^{l+p}$ глобально изометрично единичной сфере.

Для доказательства теоремы 3 осталось лишь доказать случай, когда $\gamma_{2 / F} \leqslant 1$ и $p<-1 / 2+\sqrt{1 / 4+l-1}$. По теореме $2 M^{l+p}$ глобально изометрично единичной сфере. Для компактных поверхностей в сферическом пространстве, у которых $\gamma_{2 / F} \leqslant 1$, доказано, что они являются большими сферами при $p \leqslant l / 5[1]$. Это будет, когда

$$
-\frac{1}{2}+\sqrt{\frac{1}{4}+l-1} \leqslant \frac{l}{5}
$$

Это неравенство выполняется при $l \geqslant 19$.

Харьковский государственный университет Поступило

Исправленный вариант

28.02 .95

\section{СПИСОК ЦИТИРОВАННОЙ ЛИТЕРАТУРЫ}

[1] Борисенко А. А. Об экстремальных свойствах компактных параболических поверхностей в римановом пространстве // Матем. сб. 1987. Т. 133(175). № 1. C. $112-126$.

[2] Борисенко А. А. О внешне геометрических свойствах параболических поверхностей и топологических свойствах седловых поверхностей в симметрических пространствах ранга 1 // Матем. сб. 1981. Т. 116(158). №3. С. 440-457.

[3] Эйзенхарт Л. Риманова геометрия. М.: ИЛ, 1948.

[4] Ferus D. Totally geodesic foliations // Math. Ann. 1970. V. 88. № 4. P. 313-317.

[5] Frankel T. Manifolds with positive curvature // Pacif. J. Math. 1961. V. 11. P. $165-174$.

[6] Топоногов В. А. Некоторые экстремальные теоремы римановой геометрии // Сиб. матем. ж. 1967. Т. 10. №2. С. 459-467. 